\title{
All or nothing at Fermilab
}

\author{
These days, it takes a brave laboratory to hitch its future to the construction of a brand-new particle \\ accelerator - but Fermilab has elected to do just that.
}

t t is no secret that high-energy physics faces some immense longterm challenges. Progress relies on the study of particles' behaviour at ever-higher energies, which requires larger machines, and more money to build them. The appetite of governments for such investments has waned in recent years.

These issues are well illustrated by the predicament of the Fermi National Accelerator Laboratory near Chicago. As reported on page 728 , Fermilab, the last remaining laboratory in the United States devoted to high-energy physics, is making a daring push to host a new machine, the International Linear Collider (ILC).

The ILC is an electron-positron accelerator that promises to provide further data on the Higgs boson and other particles that physicists hope to discover at CERN, Europe's premier high-energy laboratory, outside Geneva. If Fermilab builds the ILC, it will regain its position at the forefront of international science; failure could lead to staff reductions and intellectual atrophy. But landing the machine won't be easy: Fermilab will need the assurance of largescale financial support from the US Department of Energy, and the backing of the international physics community.

By choice or necessity, other high-energy physics centres around the world are ensuring that they will not have to rely on the construction of new accelerators. The Stanford Linear Accelerator Center in California, until recently the only other US lab largely devoted to high-energy physics, is reinventing itself as a 'photon science' centre - researchers will use X-rays from its accelerators to study everything from materials to proteins. Similarly, DESY, Germany's primary high-energy physics facility, near Hamburg, is building a billion-euro 'free electron' laser. And the Japanese high-energy physics laboratory KEK is building a neutron source for materials research and structural biology. They are all retaining high-energy physicists, but are not staking their fate on the next big machine.

That leaves CERN as the only other lab left as dependent on highenergy physics as Fermilab. CERN's immediate outlook is bright, with the Large Hadron Collider (LHC) due to enter service in 2008. But its secondary activities have been depleted by cost overruns on LHC construction, and in the very long run - after LHC's scope is exhausted - its future is as uncertain as Fermilab's.

High-energy physics has always drawn its focus from places such as Fermilab and CERN. They have served as a location for machines, a gathering point for the community, and a base for political support. Today, there are signs that this is changing. The Internet and the Grid - a powerful distributed-computing system now well under development are making it easier for university researchers to work on the latest data remotely. Physicists who travel to CERN, for example, won't have access to much more information from the LHC than those back at the universities.
"Other high-energy physics labs are ensuring that they will not have to rely on the construction of new accelerators."
In this interconnected world, the role of the larger centres will evolve. Fermilab's stand in seeking to retain its identity by hosting the linear collider is a bold one. Physicists are at the beginning of an exhaustive international consultation to determine the best design and site for the collider, and the lab will gain a significant advantage by moving aggressively early in the game. But, ultimately, it will require a herculean effort to marshal the political support needed to build the machine, at Fermilab or anywhere else.

\section{Kyoto - the sequel}

\section{Better knowledge of the carbon cycle could provide a basis for future climate-change agreements.}

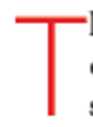
he Kyoto Protocol has only just come into force, but its 2012 expiration date already looms large on the horizon. A successor agreement faces some formidable political obstacles. But negotiators should put these to one side for now and ask themselves this: what kind of agreement is best justified by our current knowledge of climate science?

Negotiations on Kyoto II are due to begin at the eleventh meeting of the parties to the United Nations Framework Convention on Climate Change in Montreal in November. Climate science, and the tools that researchers can use to detect global change, have advanced significantly since 1997, when the Kyoto Protocol was negotiated.
These improvements must be considered when laying the basis for what comes after 2012.

One important advance has been the study of global carbon cycles - the large-scale carbon fluxes between the biosphere, the atmosphere and the oceans. Siberia, for example, is likely to undergo significant changes in vegetation, wildfire frequency and permafrost abundance, which all have a substantial impact on global carbon transfers (see page 732). Similar changes are being observed in other terrestrial ecosystems, including tropical rainforests and deserts.

Meanwhile, better satellite sensors, in combination with other remote-sensing techniques, provide ever more accurate data about biomass changes on the ground. The Siberian work has demonstrated that it is possible, in principle, to reliably determine carbon flux to and from Earth's main carbon reservoirs.

In a few years' time, a comprehensive, global carbon accounting system, incorporating natural carbon fluxes between the ground, the oceans and the atmosphere, as well as anthropogenic emissions, 